May 1993

OUTP 93-10-P

\title{
HALF-STRING APPROACH TO CLOSED STRING FIELD THEORY
}

\author{
F. Anton, A. Abdurrahman' \\ Department of Theoretical Physics, University of Oxford \\ 1 Keble Road, Oxford, OX1 3NP, U.K. \\ and \\ J. Bordest \\ Departament de Física Teórica, Universitat de Valencia \\ Dr. Moliner 50, E-46100, Burjassot, Spain.
}

\begin{abstract}
In this letter we present an operator formalism for Closed String Field Theory based on closed half-strings. Our results indicate that the restricted polyhedra of the classical non-polynomial string field theory, can be represented as traces of infinite matrices, with operator insertions that reparametrise the half-strings.
\end{abstract}

PACS numbers:11.17.+y

\footnotetext{
${ }^{1}$ Also at Mathematical Institute, 24-29, St. Giles, Oxford, OX1 3LB, U.K.

${ }^{2}$ Also at IFIC, Centro Mixto Universitat de Valencia-CSIC
} 
The problem of formulating a covariant closed string field theory proved to be surprisingly difficult. A straight forward extension of Witten's open string field theory [1] to closed strings does not satisfy gauge invariance [2] and it fails to reproduce the correct dual scattering amplitudes [3, [4].

It is now well established that a consistent closed string field theory, is necessarily nonpolynomial[5]. At the classical level, the interaction terms are the so-called restricted polyhedra [6, 7, 8], where the contact interactions are given by the patterns of string overlaps on the surface of a sphere having always three edges at each vertex. For the N-string scattering one has an $\mathrm{N}$-faced polyhedron for which the $\mathrm{N}$ closed strings correspond to the faces, and are glued together across the edges. The lengths of the edges play the role of the modular parameters. They are restricted by $(i)$, the sum of the lengths of the edges of any face equals $2 \pi$ (corresponding to the fact that all strings are taken to have the same $\sigma$-length equal to $2 \pi$ ), and (ii), any closed path surrounding two or more faces on a polyhedron has length larger than or equal to $2 \pi$.

On the other hand, based on the strong analogies between Yang-Mills theory and Witten's open string field theory, it was first suggested in 9, 10] and proved rigorously in [11, 12, 13], that physical open strings can be viewed as infinite matrices. If one breaks the open string into two pieces, the string field $\Psi$, can be treated as a functional of the two "half-strings", which play the role of the row and column indices, and a function of the mid-point. In particular the open string 3-vertex is represented as a trace[11, 12, 13]. This trace can be generalized to represent any $\mathrm{N}$-string $(N \geq 3)$ tree level scattering amplitude[15]:

$$
A_{N}=\int_{-\infty}^{\infty} \frac{d \lambda_{1} \cdots d \lambda_{N}}{S L(2, \Re)} \operatorname{Tr}\left(\exp \left(\lambda_{1} M\right) A_{1} \cdots \exp \left(\lambda_{N} M\right) A_{N}\right) .
$$

The operator $M$ is the generator of infinitesimal shifts of the mid-point of the string, so in fact we are shifting this point to every possible position.

In an analogous construction for closed strings one brakes the closed string into two pieces, therefore singling out two points, and treats each of the remaining halves as labeling matrix indices. Shifting these two points around corresponds to varying the lengths of the basic overlaps of the strings. In other words, one is varying the lengths of the edges of the polyhedra. Using a functional approach it has been proved in [16], that the analogous of eq. (1) for closed strings gives the correct dual amplitudes. Alternatively, one could restrict the region of integration in such a way that the restricted polyhedra are obtained. For the 3-vertex there are no modular parameters, so the Witten vertex should work. For higher vertices the proper region of integration has to be found in such a way that the moduli spaces of the field theory are covered correctly.

In this letter we present an oscillator construction for orbital degrees of freedom of the closed strings based on half-string coordinates (the ghost degrees of freedom will be treated elswere). We will find that the correspondence between the half-string and the full-string descriptions is non-singular. As examples we 
calculate explicitly the 1 and the 2-vertices, and we recover the correct vertex for the scattering of three tachyons. We finish this letter by showing how a vertex with no operator insertions obtained by sewing two vertices, satisfies the "gluing and resmoothing theorem" of [17 in a trivial way. Our approach will follow closely the approach of references [11, 114, 12] and [13].

Half-string coordinates. The boundary condition satisfied by the closed string is (the space-time index is not written, and the length of the strings is taken to be $\pi$ as opposed to $2 \pi)$ :

$$
X(\sigma, \tau)=X(\sigma+\pi, \tau) .
$$

The general solution to the string equations of motion compatible with (21) at $\tau=0$, can be written as

$$
X(\sigma, 0)=x_{0}+\frac{1}{\sqrt{2}} \sum_{n \geq 1}\left[x_{n} \cos 2 n \sigma+y_{n} \sin 2 n \sigma\right],
$$

where we have introduced the oscillators

$$
\begin{aligned}
& x_{n}=\frac{i}{\sqrt{2} n}\left(\alpha_{n}-\alpha_{-n}+\widetilde{\alpha}_{n}-\widetilde{\alpha}_{-n}\right)=x_{n}^{\dagger}, \\
& y_{n}=\frac{1}{\sqrt{2} n}\left(-\alpha_{n}-\alpha_{-n}+\widetilde{\alpha}_{n}+\widetilde{\alpha}_{-n}\right)=y_{n}^{\dagger} .
\end{aligned}
$$

The oscillator modes $\alpha$ and $\widetilde{\alpha}$, satisfy the well known commutation relations

$$
\left[\alpha_{n}, \alpha_{m}\right]=\left[\widetilde{\alpha}_{n}, \widetilde{\alpha}_{m}\right]=m \delta_{n+m} ; \quad\left[\alpha_{n}, \widetilde{\alpha}_{m}\right]=0 .
$$

The Fock space of the theory is constructed by acting on the vacuum $\mid 0>$, with the creation operators $\alpha_{-n}$ and $\widetilde{\alpha}_{-n}$, (with $n \geq 1$ ).

There are several possible boundary conditions for the half string coordinates. The choice we make, will determine the commutation relations of the half-string oscillator modes after quantization and therefore the half-string Fock spaces. The correct choice of boundary conditions is the one that isolates completely the motion of the two points $X\left(\sigma=\frac{\pi}{2}\right)$ and $X(\sigma=0)$, where we break the string. This is so that there is a one-to-one correspondence between the two descriptions (which in general is given by an non-singular infinite dimensional matrix). The simplest choice is the one depicted in figure (1), where we joined the string points at $\sigma=\frac{\pi}{2}$, and $\sigma=0$ with a straight line, and the half string coordinates $\chi^{r}(\sigma)$, $r=1,2$, are defined as the distances between this axis and the string. One has:

$$
\chi^{r}(\sigma, \tau)= \begin{cases}X(\sigma, \tau)-\frac{2}{\pi}\left(\frac{\pi}{2}-\sigma\right) X(0, \tau)-\frac{2 \sigma}{\pi} X\left(\frac{\pi}{2}, \tau\right) & \text { if } \mathrm{r}=1, \\ X(\pi-\sigma, \tau)-\frac{2}{\pi}\left(\frac{\pi}{2}-\sigma\right) X(0, \tau)-\frac{2 \sigma}{\pi} X\left(\frac{\pi}{2}, \tau\right) & \text { if } \mathrm{r}=2 .\end{cases}
$$

where $\sigma \in\left[0, \frac{\pi}{2}\right]$. The boundary conditions are $\chi^{r}(0)=0=\chi^{r}\left(\frac{\pi}{2}\right)$, and they imply a Fourier decomposition (at $\tau=0$ ) in terms of even sine modes only:

$$
\chi^{r}(\sigma)=\sum_{n \geq 1} \chi_{n}^{r} \sin 2 n \sigma,
$$




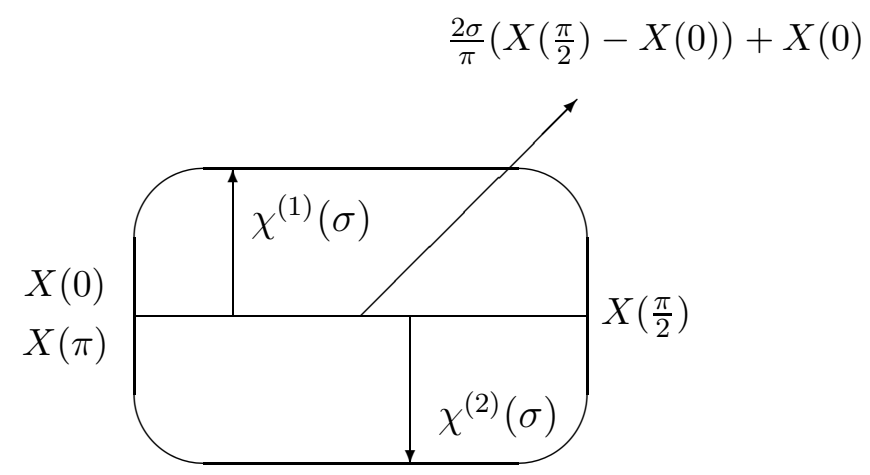

Figure 1: Definition of the Half-String Coordinates

with inverse:

$$
\chi_{n}^{r}=\frac{4}{\pi} \int_{0}^{\frac{\pi}{2}} d \sigma \chi^{r}(\sigma) \sin 2 n \sigma .
$$

Let us define the following quantities in the space of "even-odd oscillators":

$$
\mathbf{A}_{n}^{r}=\left(\begin{array}{c}
A_{2 n}^{r} \\
A_{2 n-1}^{r}
\end{array}\right) ; \quad \mathbf{B}_{n}=\left(\begin{array}{c}
B_{2 n} \\
B_{2 n-1}
\end{array}\right)
$$

Where $A(B)$ is any half-string (full-string) vector. We also define the matrix:

$$
\mathbf{B}_{n, m}=\left(\begin{array}{cc}
\left(\frac{2 m-1}{2 n}\right) B_{2 n, 2 m-1} & 0 \\
0 & \left(\frac{2 m}{2 n-1}\right) B_{2 n-1,2 m}
\end{array}\right),
$$

(bold-faced quantities will be used to represent vectors or matrices in this "evenodd" space). $B_{m, n}$ is given by:

$$
B_{m, n}=\frac{1}{\pi}\left(\frac{2 n}{n^{2}-m^{2}}\right) .
$$

Then one can express $\chi_{n}^{r}$ as:

$$
\boldsymbol{\chi}_{n}^{r}=-\sqrt{2} \sum_{m \geq 1} \mathbf{B}_{n, m} \mathbf{u} \mathbf{x}_{m}+\frac{(-)^{r+1}}{\sqrt{2}} \mathbf{y}_{n} ; \quad \mathbf{u}=\left(\begin{array}{cc}
0 & 1 \\
1 & 0
\end{array}\right)
$$

The matrix $\mathbf{B}_{n, m}$ is non-singular. Indeed it can be checked that:

$$
\left(\mathbf{B}^{-1}\right)_{m, n}=-4\left(\begin{array}{cc}
\left(\frac{2 n}{2 m-1}\right)^{2} B_{2 m-1,2 n} & 0 \\
0 & B_{2 m, 2 n-1}
\end{array}\right) .
$$


Therefore there is an invertible relation between the full-string oscillators $x_{n}$ and $y_{n}$, and the half-string modes $\chi_{n}^{r}$, the inverse of equation (13) is:

$$
\mathbf{x}_{m}=\frac{-\mathbf{u}}{2 \sqrt{2}} \sum_{n, r}\left(\mathbf{B}^{-1}\right)_{m, n} \boldsymbol{\chi}_{n}^{r} ; \quad \mathbf{y}_{n}=\frac{1}{\sqrt{2}} \sum_{r=1,2}(-)^{r+1} \boldsymbol{\chi}_{n}^{r}
$$

To complete the picture, we still need an explicit relation between the center of mass coordinate $x_{0}$, and the two points of the string that we singled out. Calling them $X_{I}=X\left(\sigma=\frac{\pi}{2}, \tau=0\right)$ and $X_{I I}=X(\sigma=0, \tau=0)$, it is straight forward to see that:

$$
x_{0}=\frac{1}{2}\left(X_{I}+X_{I I}\right)+\frac{1}{\pi} \sum_{n, r} \frac{\chi_{2 n-1}^{r}}{2 n-1} .
$$

Hence there exists a one-to-one correspondence between the half-string and the full string pictures. To establish this correspondence at the level of Fock spaces, we proceed to quantize the theory.

The quantization programme can be carried out in the usual way by interpreting the oscillator modes $\chi_{n}^{r}, X_{I}$ and $X_{I I}$ as q-operators and define their conjugate momenta as $P_{I}=-i \frac{\partial}{\partial X_{I}}, P_{I I}=-i \frac{\partial}{\partial X_{I I}}$ and $P_{n}^{r}=-i \frac{\partial}{\partial \chi_{n}^{r}}$. They satisfy

$$
\left[\chi_{n}^{r}, P_{n}^{s}\right]=i \delta^{r, s} \delta_{n, m}, \quad\left[X_{I}, P_{I}\right]=i, \quad\left[X_{I I}, P_{I I}\right]=i
$$

The corresponding momenta for the full string are given by $P_{n}=-i \frac{\partial}{\partial x_{n}}, \widetilde{P}_{n}=$ $-i \frac{\partial}{\partial y_{n}}$ and $P=-i \frac{\partial}{\partial x_{0}}$.

The transformation rules between the half and the full-string momenta can be easily worked out using the chain rule to give:

$$
\mathbf{P}_{m}^{r}=-\frac{\mathbf{u}}{2 \sqrt{2}} \sum_{n \geq 1}\left(\mathbf{B}^{-1}\right)_{n, m} \mathbf{P}_{n}+\frac{(-)^{r+1}}{\sqrt{2}} \widetilde{\mathbf{P}}_{m}+\frac{\mathbf{e}_{2}}{\pi} \frac{P}{(2 m-1)}
$$

and

$$
\frac{1}{2} P=P_{I}+P_{I I}
$$

Here $\mathbf{e}_{2}$ is the unit vector $(0,1)^{T}$. These equations can be inverted to solve for $P_{m}$

$$
\mathbf{u} \mathbf{P}_{m}=-\sqrt{2} \sum_{n, r} \mathbf{B}_{n, m} \mathbf{P}_{n}^{r}+\frac{\mathbf{e}_{2}}{\sqrt{2}} P \quad \text { and } \quad \widetilde{\mathbf{P}}_{m}=\sum_{r} \frac{(-)^{r+1}}{\sqrt{2}} \mathbf{P}_{m}^{r}
$$

$P$ is recovered by multiplying the first of eqs. (19) by $\frac{1}{2 k-1}$, and summing over $r=1,2$ and odd $n \geq 1$ :

$$
P=\frac{4}{\pi} \sum_{r, k} \frac{1}{2 k-1} P_{2 k-1}^{r} .
$$

Relation (18) may seem somewhat obscure at this point. It can be better understood at the classical level in the following way: from equation (7) we can get 
an expression for the string Lagrangian, from which $P_{I}+P_{I I}=\int_{0}^{\frac{\pi}{2}} \sum_{r} P^{r}$, follows as a primary constraint on the system. The total momentum of the string is $P_{\text {total }}=P_{I}+P_{I I}+\int_{0}^{\frac{\pi}{2}} \sum_{r} P^{r}=2\left(P_{I}+P_{I I}\right)$, in agreement with equation (18). This identification means that $2\left(P_{I}+P_{I I}\right)$ corresponds to the translational mode of the string. This will become clear since integration over the combination $\frac{1}{2}\left(X_{I}+X_{I I}\right)$ gives rise to the conservation of momentum in the vertices.

Let us pause for a moment too see the meaning of this. Traditionally, people have been bias against using half-strings to describe closed strings because this would produce a bilocal theory. However from eqs. (16) and (18) we see that in fact the string fields only depend on the two preferred points through the combination $X_{I}+X_{I I}$.

The full-string Fock space is build up by acting on the vacuum $\mid 0>$ with the creation operators:

$$
a_{m}^{\dagger}=\frac{1}{\sqrt{2 m}}\left(\alpha_{-m}+\widetilde{\alpha}_{-m}\right) ; \quad \widetilde{a}_{m}^{\dagger}=\frac{i}{\sqrt{2 m}}\left(\alpha_{-m}-\widetilde{\alpha}_{-m}\right)
$$

corresponding to the modes $x_{n}$ and $y_{n}$ respectively. The creation and annihilation operators for the half-string defined as:

$$
\beta_{n}^{r \dagger}=i \frac{\sqrt{n}}{2} \chi_{n}^{r}+\frac{1}{\sqrt{n}} P_{n}^{r} ; \quad \beta_{n}^{r}=-i \frac{\sqrt{n}}{2} \chi_{n}^{r}+\frac{1}{\sqrt{n}} P_{n}^{r},
$$

are related to the full string oscillator modes by

$$
\mathbf{u} \mathbf{a}_{m}=\sum_{n \geq 1}\left(\mathbf{A}_{m n}^{(+)} \mathbf{b}_{n}^{(+) \dagger}+\mathbf{A}_{m n}^{(-)} \mathbf{b}_{n}^{(+)}\right)+\frac{\mathbf{e}_{2} P}{2 \sqrt{m}} ; \quad \text { and } \quad \widetilde{\mathbf{a}}_{m}=\mathbf{b}_{m}^{(-)}
$$

where $b_{n}^{ \pm}=\frac{1}{\sqrt{2}}\left(\beta_{n}^{(1)} \pm \beta_{n}^{(2)}\right)$, and we have defined the following matrices:

$$
\begin{gathered}
\mathbf{A}_{m, n}^{ \pm}=\left(\begin{array}{cc}
-N_{m, n}^{ \pm} & 0 \\
0 & -M_{m, n}^{ \pm}
\end{array}\right) \\
M_{m n}^{ \pm}=\left(\frac{2 m}{2 n-1}\right)^{\frac{1}{2}}\left[B_{2 n-1,2 m} \pm B_{2 m, 2 n-1}\right]
\end{gathered}
$$

and

$$
N_{m n}^{ \pm}=\left(\frac{2 n}{2 m-1}\right)^{\frac{1}{2}}\left[\left(\frac{2 m-1}{2 n}\right) B_{2 n, 2 m-1} \pm\left(\frac{2 n}{2 m-1}\right) B_{2 m-1,2 n}\right] .
$$

Notice that the matrix $M^{ \pm}$, coupling the odd oscillators already appeared in [11, 14] for the open bosonic string. Next, we go on to develop a relation between the full string vacuum $\mid 0>$, and the two half-string vacua $\mid 0>_{1}$ and $\mid 0>_{2}$ corresponding to the two half-strings. They are defined in the usual way:

$$
\beta_{m}^{r} \mid 0>_{r}=0
$$


Repeated action of the creation operators $\beta_{m}^{r \dagger}$, on these vacua give the Fock spaces corresponding to each half-string. We propose the standard ansatz

$$
\left|0>=\exp \left(\frac{-1}{2} \mathbf{b}_{n}^{(+) \dagger} \boldsymbol{\xi}_{n m} \mathbf{b}_{m}^{(+) \dagger}\right)\right| 0>_{1} \mid 0>_{2}
$$

Notice that only the plus combination appears in the exponential. This is because $\widetilde{a}_{n}$ is expressed only in terms of $b_{n}^{(-)}$, and hence it annihilates the vacuum trivially. The action of $a_{n}$ fixes $\boldsymbol{\xi}$ to be:

$$
\boldsymbol{\xi}=\mathbf{A}^{(-)-1} \mathbf{A}^{(+)}=\left(\begin{array}{cc}
\psi & 0 \\
0 & \varphi
\end{array}\right) .
$$

The tachyon state is obtained by inserting the factor $e^{i P x_{0}}$ in (28). One can verify that this state in the half-string representation is an eigenstate of the momentum operator with eigenvalue $P$, when expressed in the half-string language.

The Hilbert space $\mathcal{H}$ of the closed full string is spanned by linear combinations of the state vectors of the form $\prod a_{n}^{\dagger}, \widetilde{a}_{n}^{\dagger} \mid 0>$. The one-to-one relation between full and half-string modes, together with equation (28) imply that the Hilbert space of the full string is contained in the completion of the tensor product $\overline{\mathcal{H}}=\overline{\mathcal{H}_{1} \otimes \mathcal{H}_{2} \otimes \mathcal{H}_{M}}$.

Matrix representations and verices. In order to compute the half-string matrix representing a string state with arbitrary ocupation nubers, it is useful to use the coherent state:

$$
\mid \vec{\lambda}, \overrightarrow{\widetilde{\lambda}} ; p)=e^{i p x_{0}} \exp \left(\lambda \cdot a^{\dagger}+\tilde{\lambda} \cdot \widetilde{a}^{\dagger}\right) \mid 0>
$$

Also one introduces the half-string states:

$$
\left|n_{i}^{r}>=\prod_{i=1}^{\infty} \frac{1}{\sqrt{n}_{i}^{r} !}\left(\beta_{i}^{r}\right)^{\dagger n_{i}^{r}}\right| 0>_{r}
$$

in terms of which, the matrix representing the functional for any state is defined by:

$$
\left.[A]_{n_{i}^{1}}^{n_{i}^{2}}=(-)^{\sum_{i \geq 1} n_{i}^{2}}<n_{i}^{1} ; n_{i}^{2} \mid \lambda, \widetilde{\lambda} ; p\right) .
$$

The factor $(-)^{\sum_{i \geq 1} n_{i}^{2}}$ takes into account that the normalization for $\chi^{2}$ is reversed.

After a long calculation we find the following expression for the matrix (32):

$$
\begin{aligned}
{[A]_{n_{i}^{1}}^{n_{2}^{2}}=} & C(\lambda ; p) e^{\frac{i p}{2}\left(X_{I}+X_{I I}\right)} \prod_{i=1}^{\infty} \frac{1}{\sqrt{n_{i}^{1} ! n_{i}^{2} !}}\left(\frac{-1}{\sqrt{2}} D_{i}^{-}\right)^{n_{i}^{1}}\left(\frac{1}{\sqrt{2}} D_{i}^{+}\right)^{n_{i}^{2}} \times \\
& \left.\exp \left(\frac{-1}{2} \mathbf{v}^{T} \boldsymbol{\xi} \mathbf{v}\right)\right|_{v=0}
\end{aligned}
$$


where we have defined the quantities:

$$
\begin{aligned}
C(\lambda ; p)= & \exp \left(-\frac{1}{2} p^{2} \mathbf{k}^{T}(\mathbf{1}+\boldsymbol{\xi}) \mathbf{k}+p \boldsymbol{\lambda}^{T}\left(\mathbf{A}^{-}-\mathbf{A}^{+} \boldsymbol{\xi}\right) \mathbf{k}\right. \\
& \left.+\frac{1}{2} \boldsymbol{\lambda}^{T}\left(\mathbf{A}^{-}-\mathbf{A}^{+} \boldsymbol{\xi}\right) \mathbf{A}^{(+) T} \boldsymbol{\lambda}\right) . \\
& \boldsymbol{\lambda}_{i}=\left(\begin{array}{c}
\lambda_{i}^{\prime} \\
\lambda_{i}
\end{array}\right) ; \quad \widetilde{\boldsymbol{\lambda}}_{i}=\left(\begin{array}{c}
\widetilde{\lambda}_{i} \\
\widetilde{\lambda}_{i}^{\prime}
\end{array}\right),
\end{aligned}
$$

(lambdas with a prime are those that couple to the odd modes of the creation operators in equation (30)).

$$
D_{i}^{ \pm}=\rho_{i} \pm \tilde{\lambda}_{i}+\frac{d}{d v_{i}} ; \quad \mathbf{k}_{i}=\frac{-1}{\pi \sqrt{2}}\left(\begin{array}{c}
0 \\
(2 i-1)^{-\frac{3}{2}}
\end{array}\right) .
$$

$v$ is a dummy variable and $\rho$ is given by

$$
\rho_{i}^{T}=\left[\boldsymbol{\lambda}^{T}\left(\mathbf{A}^{-}+\mathbf{A}^{+} \boldsymbol{\xi}\right)\right]_{i}-p\left[\mathbf{k}^{T}(\mathbf{1}+\boldsymbol{\xi})\right]_{i} .
$$

The coherent state matrix (33) can be used to construct the vertices in the halfstring language as:

$$
V_{N}=\frac{1}{2} \int d\left(X_{I}+X_{I I}\right) \int_{D_{N}} \frac{d \lambda_{1} \cdots d \lambda_{N}}{S L(2, \Re)} \operatorname{Tr}\left(\exp \left(\lambda_{1} M\right) A_{1} \cdots \exp \left(\lambda_{N} M\right) A_{N}\right) .
$$

The operator $M=L_{1}-L_{-1}+\widetilde{L}_{1}-\widetilde{L}_{-1}$ is the element of the $S L(2 ; \Re)$ subalgebra of the Virasoro algebra that reparametrises $\sigma$ in such a way as to shrink the segment between $\sigma=\frac{\pi}{4}$ and $\sigma=\frac{3 \pi}{4}$, while expanding its complement [16]. Although this operator leaves the points $\sigma=0, \pi$ and $\frac{\pi}{2}$ invariant, one is free to move these points to any other point by a rigid rotation since, in order to build the field theory, these vertices still have to be multiplied by the projection operator $P$ that ensures that condition (2) is satisfied. $D_{N}$ represents the region in moduli space such the restricted polyhedra are obtained. We postpone to a later paper its determination. For $N=1,2$ and 3 no $M$ insertions are necessary.

As examples we will compute the (integration) 1-vertex, the 2-vertex (sewing ket) and the 3 -vertex that couples three tachyons. Setting $N=1$ in equation (38), using equation (33) gives:

$$
I=\left.\delta(P) C(\lambda ; P) \exp \left(\frac{-1}{2} D^{+} D^{-}\right) \exp \left(\frac{-1}{2} \mathbf{v}^{T} \boldsymbol{\xi} \mathbf{v}\right)\right|_{v=0} .
$$

Integration over the combination $\frac{1}{2}\left(X_{I}+X_{I I}\right)$ gave rise to the momentum conservation delta. The sum over $n_{n_{i}}^{1}$ on the trace was grouped in the exponential. Using the standard techniques in Gaussian integrals we can write this as:

$$
I=C(\lambda ; P=0) \exp \left(\frac{1}{2}\left[\tilde{\boldsymbol{\lambda}}^{2}-\boldsymbol{\rho}^{T}(\mathbf{1}-\boldsymbol{\xi})^{-1} \boldsymbol{\rho}\right]\right) .
$$


It is not difficult to check that:

$$
(\mathbf{1}-\boldsymbol{\xi})^{-1}=\left(\mathbf{A}^{-}+\mathbf{A}^{+}\right)^{T} \mathbf{A}^{-},
$$

so that

$$
I=\exp \left(\frac{1}{2}\left[\tilde{\boldsymbol{\lambda}}^{2}-\boldsymbol{\lambda}^{2}\right]\right)
$$

This vertex precisely corresponds to folding the closed string along its diameter.

Setting $N=2$ in equation (38) gives

$$
V_{2}=\left.\delta\left(P_{1}+P_{2}\right) C\left(\lambda_{1} ; P_{1}\right) C\left(\lambda_{2} ; P_{2}\right) \exp \left(\frac{-1}{2} \vec{D}^{+} B \vec{D}^{-}\right) \exp \left(\frac{-1}{2} \mathbf{v}^{T} \boldsymbol{\xi} \mathbf{v}\right)\right|_{v=0} .
$$

Where as before integration over the combination $\frac{1}{2}\left(X_{I}+X_{I I}\right)$ gives rise to the momentum conservation delta, and the sum on the trace was grouped in the exponential. It is understood that the above quantities have been imbedded in two dimensional vectors or matrices. For example $\vec{p}=\left(p_{1}, p_{2}\right)$ are the momenta of the two external states, etc. The matrix $B$ is defined as

$$
B=\left(\begin{array}{ll}
0 & 1 \\
1 & 0
\end{array}\right) \text {. }
$$

In the same way as before, we can write $V_{2}$ (ignoring the momentum conservation delta) as:

$$
V_{2}=C\left(\lambda_{1} ; P_{1}\right) C\left(\lambda_{2} ; P_{2}\right) \exp \left(\frac{1}{2}\left[\tilde{\boldsymbol{\lambda}}^{T} B \widetilde{\boldsymbol{\lambda}}-\boldsymbol{\rho}^{T}(B-\boldsymbol{\xi})^{-1} \boldsymbol{\rho}\right]\right) .
$$

It is not difficult to check that:

$$
-\frac{1}{2} \boldsymbol{\rho}^{T}(B-\boldsymbol{\xi})^{-1} \boldsymbol{\rho}=-\frac{1}{2} \boldsymbol{\rho}_{1} \mathbf{A}^{+T} \mathbf{A}^{-} \boldsymbol{\rho}_{1}-\frac{1}{2} \boldsymbol{\rho}_{2} \mathbf{A}^{+T} \mathbf{A}^{-} \boldsymbol{\rho}_{2}-\boldsymbol{\rho}_{1} \mathbf{A}^{-T} \mathbf{A}^{-} \boldsymbol{\rho}_{2}
$$

so we arrive at

$$
V_{2}=\exp \left(\widetilde{\boldsymbol{\lambda}_{1}} \cdot \widetilde{\boldsymbol{\lambda}_{2}}-\boldsymbol{\lambda}_{\mathbf{1}}^{T} \cdot \boldsymbol{\lambda}_{\mathbf{2}}\right),
$$

which again can be seen to correspond to the standard result.

We now proceed to the computation of the 3 -vertex. From (38):

$$
\begin{gathered}
\quad=\delta\left(\sum_{i=1}^{3} p_{i}\right) e^{\left(a \sum_{r=1}^{3} p^{(r)^{2}}\right)} \\
\left.V_{3} \widetilde{\overrightarrow{\vec{\lambda}}}^{T} M_{1} \widetilde{\vec{\lambda}}+\vec{\lambda}^{T} M_{1}^{\prime} \vec{\lambda}+\widetilde{\vec{\lambda}}^{T} M_{2} \vec{p}+\vec{\lambda}^{T} M_{2}^{\prime} \vec{p}+\widetilde{\vec{\lambda}}^{T} M \vec{\lambda}\right] .
\end{gathered}
$$

As before we have imbedded the above quantities in the three dimensional space spanned by the three strings. The quantity $a$ and the matrices $M_{i}, M_{i}^{\prime}$ and $M$, are the same quantities that appeared in [11], with $\phi \rightarrow \boldsymbol{\xi}$ and $k_{n} \rightarrow \mathbf{k}_{n}$. 
Setting $\lambda=\widetilde{\lambda}=0$ in the above expression, one recovers the interaction of three tachyons ${ }^{3}$, namely

$$
V_{3}^{\text {Tachyons }}=\delta\left(\sum_{i=1,2,3} P_{0}^{i}\right) e^{\frac{-1}{8} \ln \left(\frac{3^{3}}{2^{4}}\right) \sum_{i=1,2,3} P_{0}^{(i) 2}} .
$$

In arriving at this expression we have used that $a=-\frac{1}{4} N_{00}^{r s}$, which can be easily established with the results of references[11, 14].

These three examples coincide with the standard expressions. However we still have to compare our higher order vertices with other vertices appearing in the literature. In [17], LeClair, Peskin and Preitschopf (LPP) define the 3-vertex through the relation:

$$
<V_{123}|| A>_{1}\left|B>_{2}\right| C>_{3}=<T^{2} h\left[\Theta_{A}\right] T h\left[\Theta_{B}\right] h\left[\Theta_{C}\right]>
$$

here $\Theta_{A}$ is the normal ordered operator that creates the state $\mid A>$ :

$$
\left|A>=\Theta_{A}\right| 0>
$$

and $T$ is an $S l(2 ; C)$ transformation such that $T^{3}=1$. Higher order vertices are defined analogously.

There are precisely these type of vertices the ones used as a starting point by Kugo and Suehiro, to construct the restricted polyhedra, and to show that the resulting theory is gauge invariant [8].

Lets start by comparing the two-point vertices in both formalisms. By definition the state $\mid V_{12}>$ (or simply $\mid V^{(2)}>$ in our language) imposes the condition $X_{1}(\sigma)=X_{2}(-\sigma)$, which is equivalent to imposing that $\alpha_{n}^{(1)}=\alpha_{-n}^{(2)}$ at the level of operators. If we write the string coordinate in terms of the complex coordinate $z=\exp (\tau+i \sigma)$ (take $\tau=0$ ), then this condition is equivalent to imposing $X_{1}(z)=X_{2}\left(\frac{1}{z}\right)$. This can be used to define the Belavin, Polyakov and Zamolodchikov (BPZ) conjugate to $\mid A>$ as

$$
<A|=<0| I\left[\Theta_{A}(0)\right]
$$

where $I(z)=\frac{1}{z}$ is a conformal map. i.e.:

$$
I\left[A\left(z, z^{*}\right)\right]=A^{\prime}\left(\frac{1}{z}, \frac{1}{z^{*}}\right) .
$$

The (BPZ) inner product is defined as:

$$
<A|B>=<0| I\left[\Theta_{A}(0)\right] \Theta_{B}(0)\left|0>=<V_{A B}\right||A>| B>.
$$

It is easy to see that the 2-vertex constructed with LPP's prescription using the $I$ defined above, corresponds exactly to our $V_{2}$.

\footnotetext{
${ }^{3}$ Recall that for closed strings $P^{2}=\frac{1}{2} P_{\text {left }}^{2}+\frac{1}{2} P_{\text {right }}^{2}=\frac{1}{2} P_{\text {open }}^{2}$.
} 
A very important property any vertex should satisfy is that the new vertex produced by the contraction of two other vertices by the conformal field theory inner product BPZ, is precisely the one which results from first sewing the corresponding two Riemann surfaces via the map $I$, and then constructing the vertex on that surface. In other words:

$$
<V_{A B E F}^{(4)}\left|=<V_{A B C}^{(3)}\right|<V_{D E F}^{(3)}|| V_{C D}^{(2)}>
$$

with

$$
<V_{1234}|| A>_{1}\left|B>_{2}\right| C>_{3} \mid D>_{4}=<T^{2} h\left[\Theta_{A}\right] T h\left[\Theta_{B}\right] I T^{2}\left[\Theta_{C}\right] I T\left[\Theta_{D}\right]>.
$$

This is the Generalized Gluing and Resmoothing Theorem (GGRT) for $N=3$ [17].

It is straight forward to show that our vertices satisfy this theorem. The proof steams out from the fact that they are written as traces, and that the transformation from half-strings to full-strings is complete.

Denote our string field matrices as $A n m=<n m \mid \Lambda)$ where the indices $n$ and $m$ refer to the left and right parts of the string, and $\Lambda$ is a short for $(\lambda, \tilde{\lambda})$. Completeness of the (orthogonal) transformations means that Parseval's identity, $I=\sum_{n m}|n ; m><n ; m|$, works both ways, and $\left.I=\int D \Lambda \mid \Lambda\right)(\Lambda \mid$. The left hand side of equation (55) can be written in our language as (summing over repeated indices):

$$
\begin{array}{r}
\left.\left.\left.V_{125}^{(3)} V_{56}^{(2) \dagger} V_{634}^{(3)}=\int D \Lambda^{5} D \Lambda^{6}<n m \mid \Lambda^{1}\right)<m k \mid \Lambda^{2}\right)<k n \mid \Lambda^{5}\right) \\
\left.\left(\Lambda^{5}\left|p q>\left(\Lambda^{6}|q p><r s| \Lambda^{6}\right)<s v\right| \Lambda^{3}\right)<v r \mid \Lambda^{4}\right) .
\end{array}
$$

Using (twice) that $\left.\int D \Lambda<k n \mid \Lambda\right)\left(\Lambda \mid p q>=\delta_{k p} \delta_{n q}\right.$ (orthogonality), we can write the above equation as

$$
\left.\left.\left.\left.V_{1234}^{(4)}=<n m \mid \Lambda^{1}\right)<m k \mid \Lambda^{2}\right)<k q \mid \Lambda^{3}\right)<q n \mid \Lambda^{4}\right),
$$

which is just the 1.h.s. of (55).

This can be generalized trivially to higher point vertices. Notice that this result is valid for both open and closed strings.

Conclusions. We have showed that closed string fields can be represented by matrices, in a very similar way as the open string ones. We only dealt with the orbital degrees of freedom, but the ghosts can also be treated with half-strings in a very similar way as in references [12, 13]. We defined the closed half-string coordinates, and from them we constructed the corresponding half-string matrices. These are written in a very similar way as the matrices corresponding to open string functionals. We constructed the vertices $V_{N}$ for $N=1,2$ and 3 explicitly, and we showed that -as for open strings- they are determined completely by the matrix $\mathbf{B}_{n m}$, which relates the full and the half string modes. This will still hold for higher order interactions. We obtained the correct vertex that couples three tachyons together and showed that our vertices satisfy the "general gluing and resmoothing theorem" of [17] in an almost trivial way. 


\section{References}

[1] E. Witten, Nucl. Phys. B268, (1986) 253.

[2] S. B. Giddins, E. Martinec, Nucl.Phys. B278 (1986) 91.

[3] M. Kaku, Phys. Rev.D38 (1988) 3052 and M. Kaku and J. Lykken, Phys. Rev.D38(1988) 3067.

[4] B. Zwiebach, Ann. Phys. 186, (1988) 111.

[5] B. Zwiebach, Nucl. Phys. B336, (1990) 185.

[6] M. Saadi and B. Zwiebach, Annals of Phys 192 (1989) 213.

[7] T. Kugo, H. Kunitomo and K. Suehiro, Phys. Lett. B226 (1989) 48.

[8] T. Kugo and K. Suehiro, Nucl. Phys. B337 (1990) 434.

[9] H.M. Chan and S.T. Tsou, Phys. Rev. D35 (1987) 2474.

[10] H.M. Chan and S.T. Tsou, Phys. Rev. D39 (1989) 555.

[11] J. Bordes, H.M. Chan, L. Nellen and S.T. Tsou, Nucl.Phys. B351 (1991) 441.

[12] A. Abdurrahman, F. Anton and J. Bordes, Nucl. Phys. To appear in Nucl. Phys. B.

[13] A. Abdurrahman, F. Anton and J. Bordes, Nucl. Phys. To appear in Nucl. Phys. B.

[14] J. Bordes, A. Abdurrahman and F. Anton, The N-String Vertices of String Field Theory. To appear.

[15] H.M. Chan, J. Bordes, S.T. Tsou, and L. Nellen, Phys. Rev. D40 (1989) 2620 .

[16] H.M. Chan, S.T. Tsou, L. Nellen and J. Bordes, Phys. Rev. D44 (1991) 1786.

[17] A. LeClair, M.E. Peskin and C.R. Preitschopf, Nucl. Phys. B317 (1989) 411. 